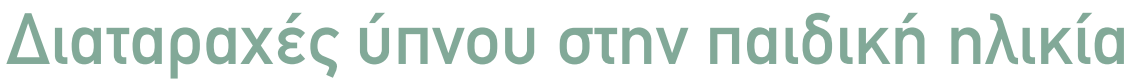

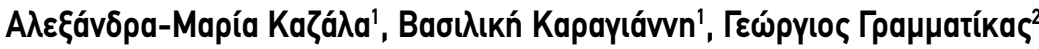

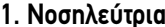

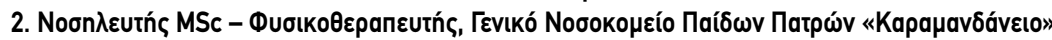

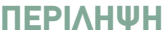

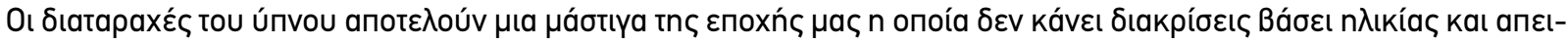

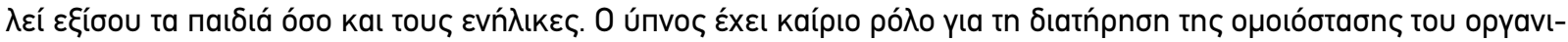

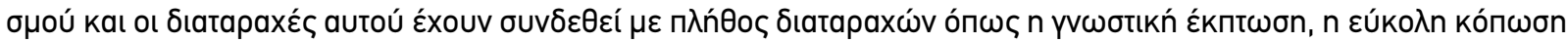

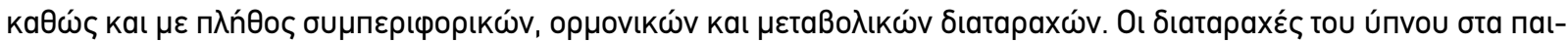

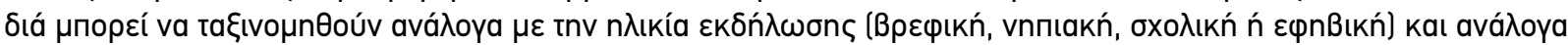

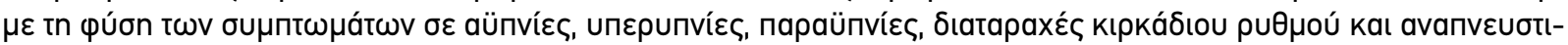

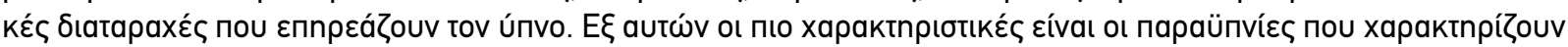

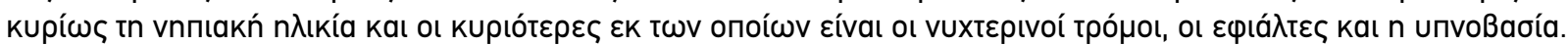

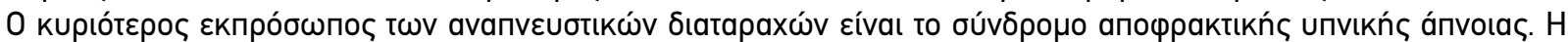

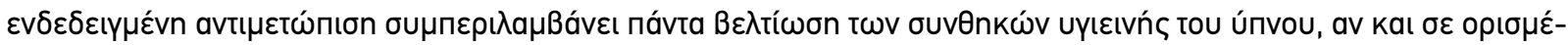

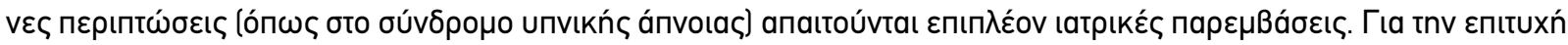

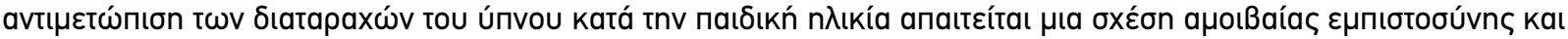

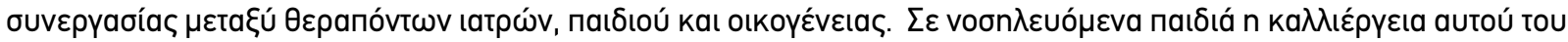

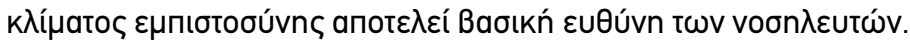

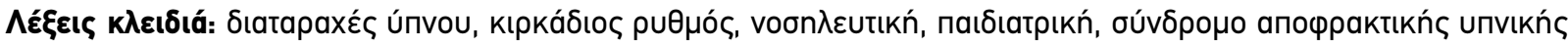
ánvolas 


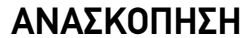

\section{BAEIKA EHMEIA:}

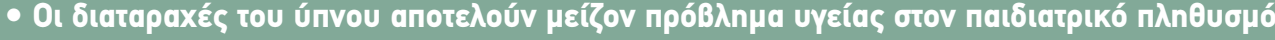

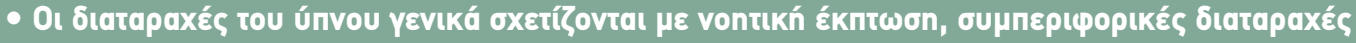

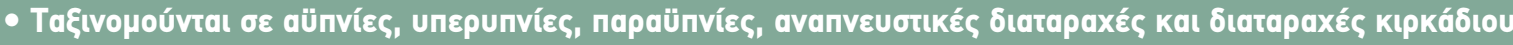
puӨpoú

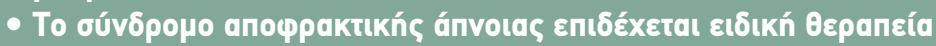

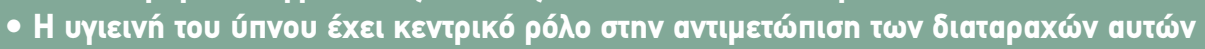

\section{EIIAГRГH}

O

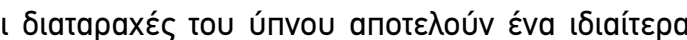

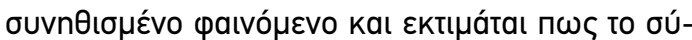

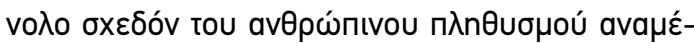

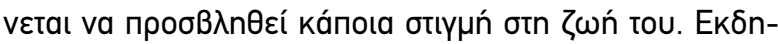

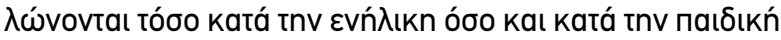

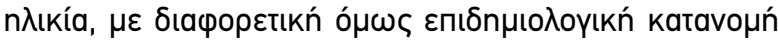

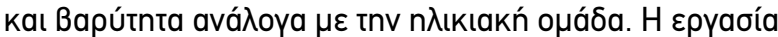

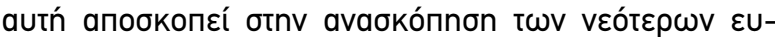

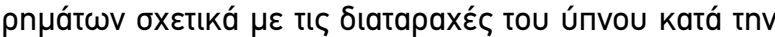

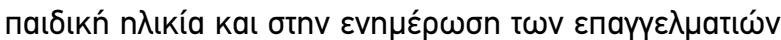

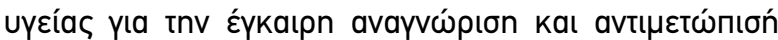

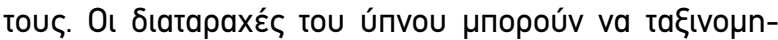

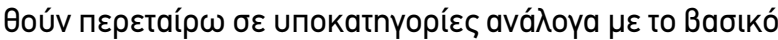

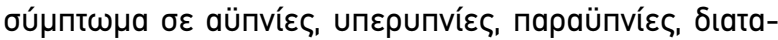

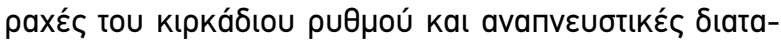

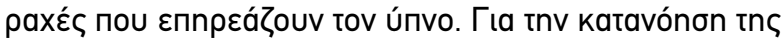

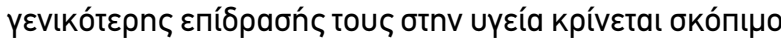

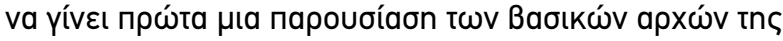

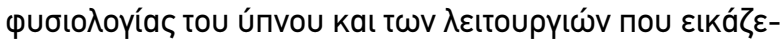

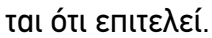

\section{Фuбtodoyía tou únvou}

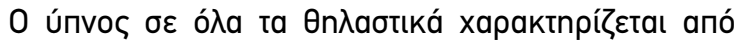

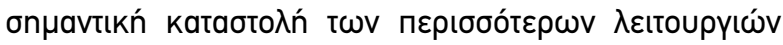

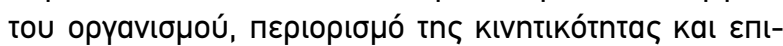

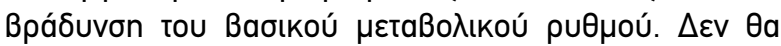

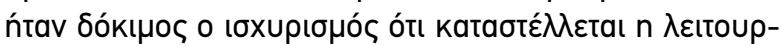

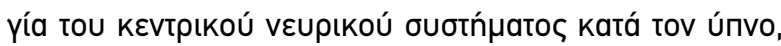

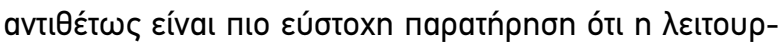

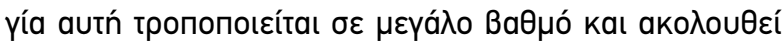

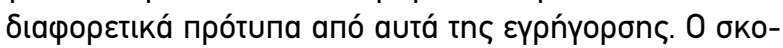

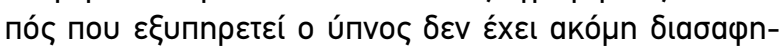

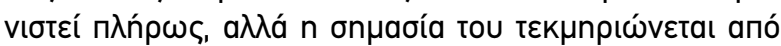

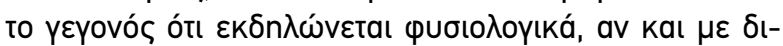

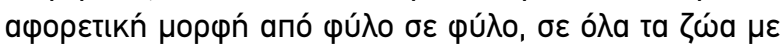

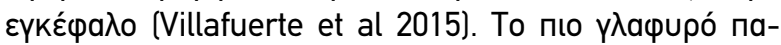

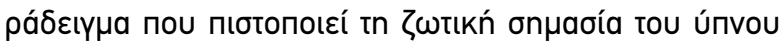

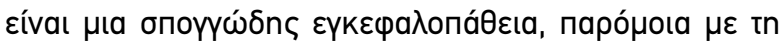

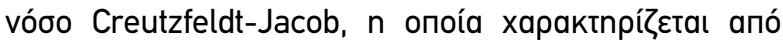

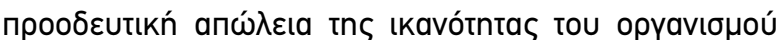

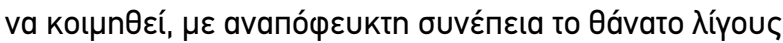

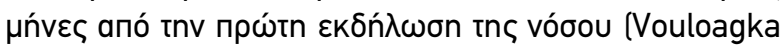
et al 2014).

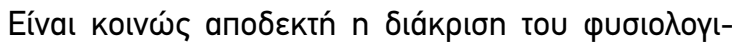

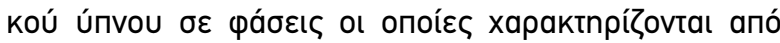

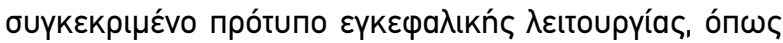

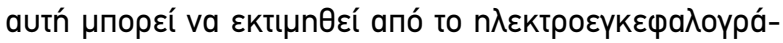

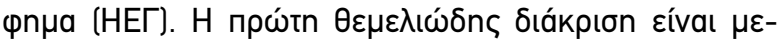

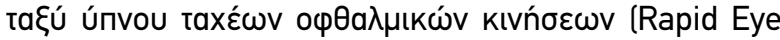

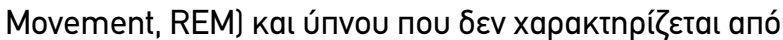

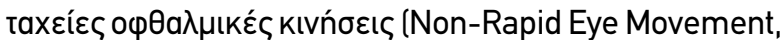

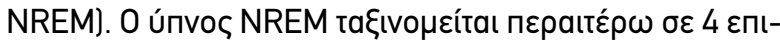

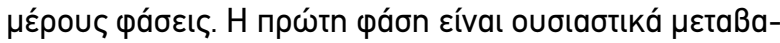

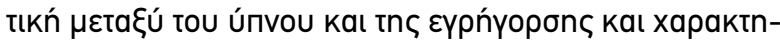

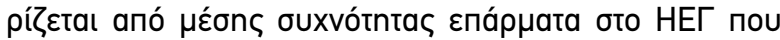

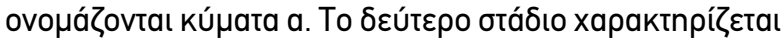

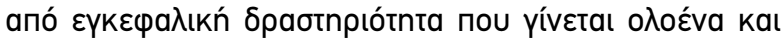

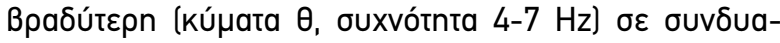

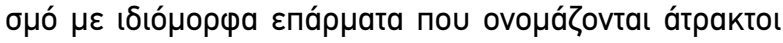

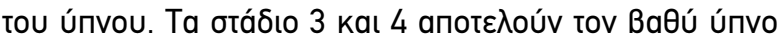

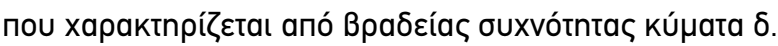

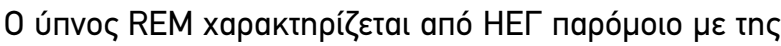

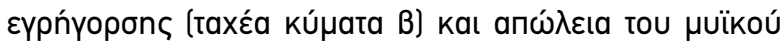

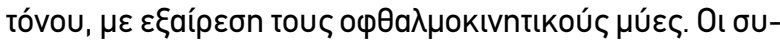

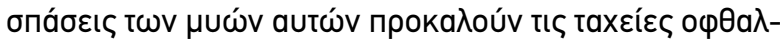

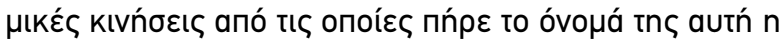
yáon tou únvou (Cirelli \& Tononi 2015).

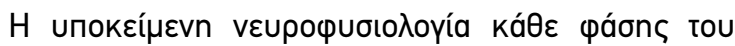

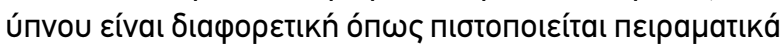

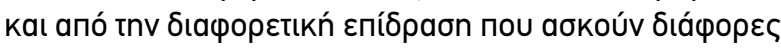

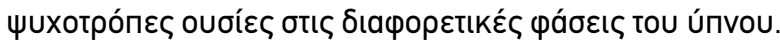

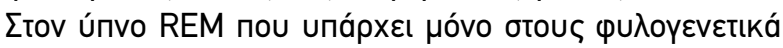

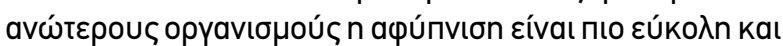

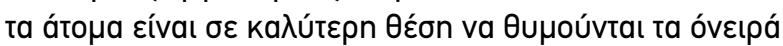

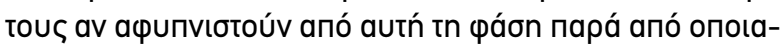

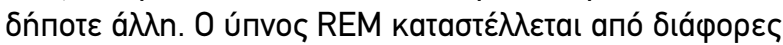

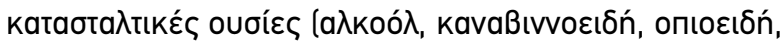

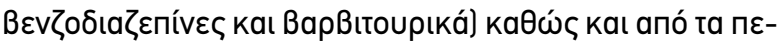

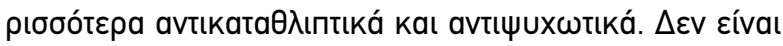




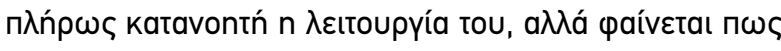

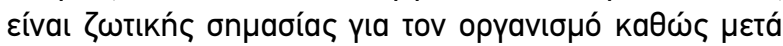

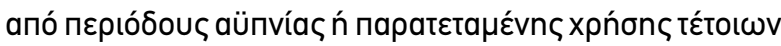

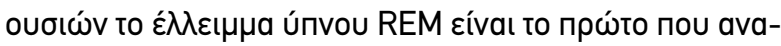

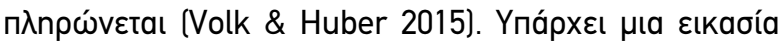

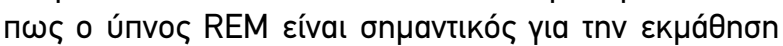

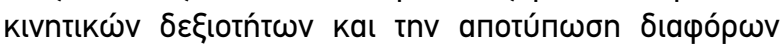

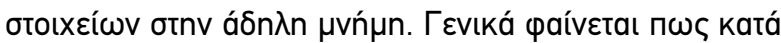

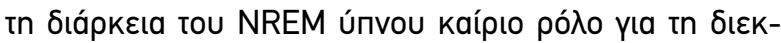

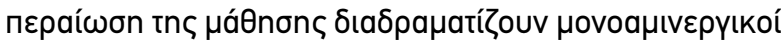

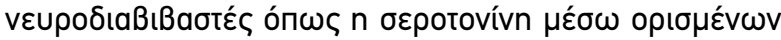

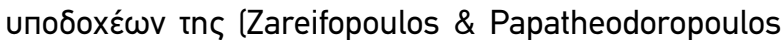

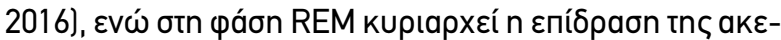

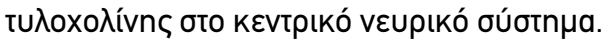

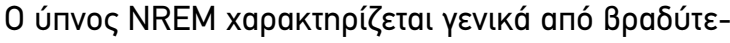

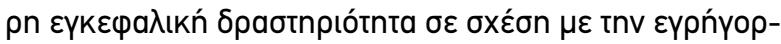

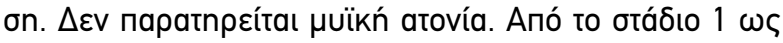

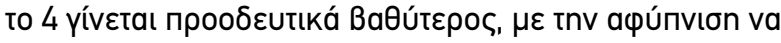

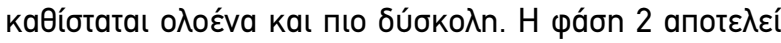

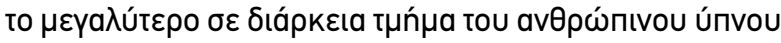

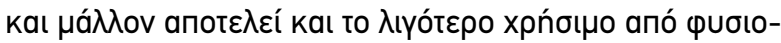

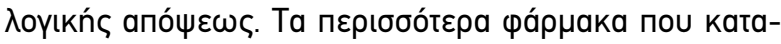

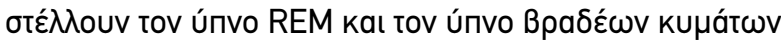

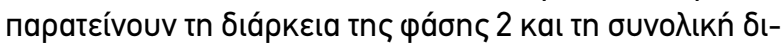

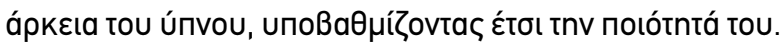

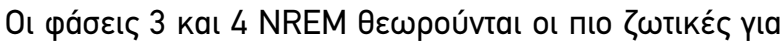

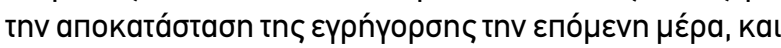

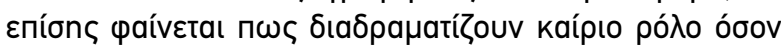

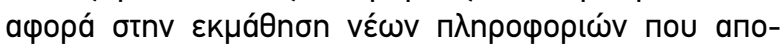

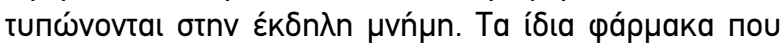

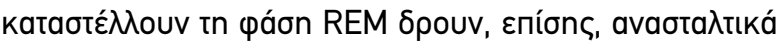

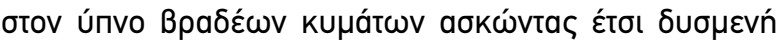

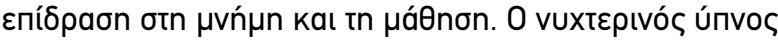

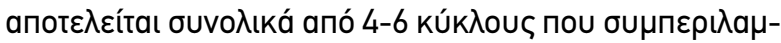

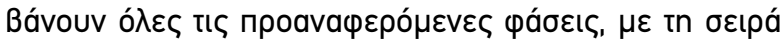
NREM2- NREM3- NREM4- NREM3- NREM2- REM-

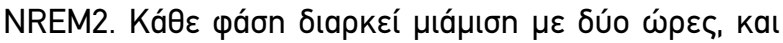

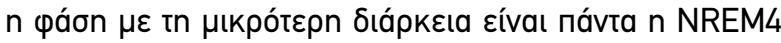
(Cirelli \& Tononi 2015).

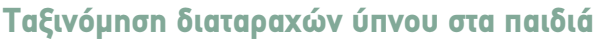

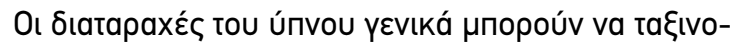

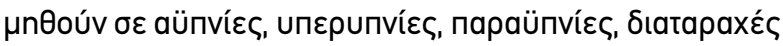

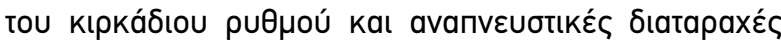

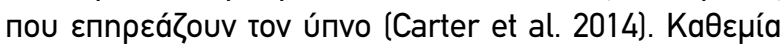

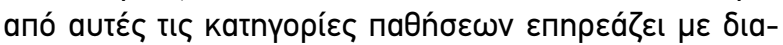

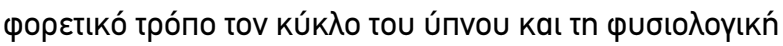

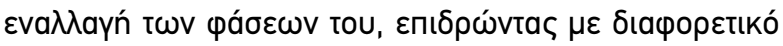

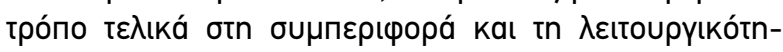

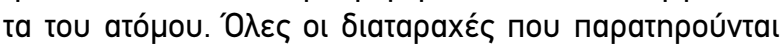

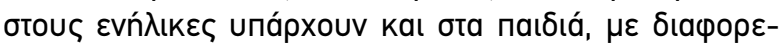

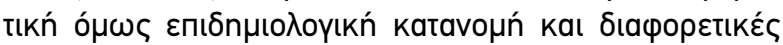

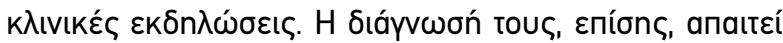

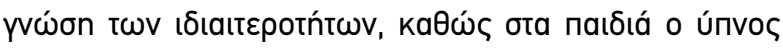

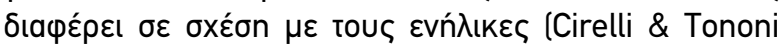

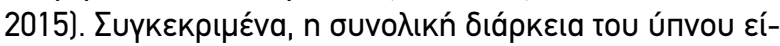

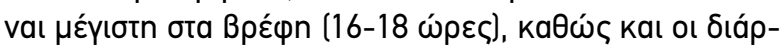

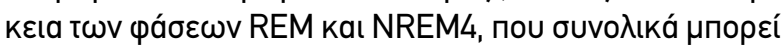

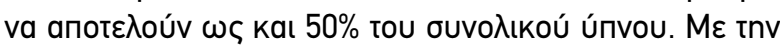

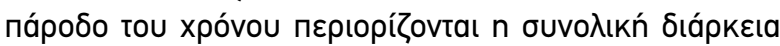

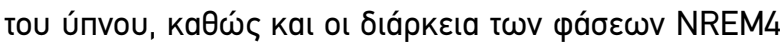

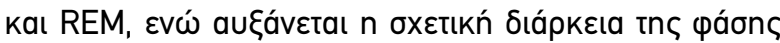

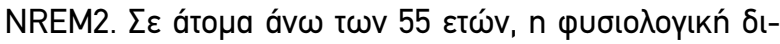

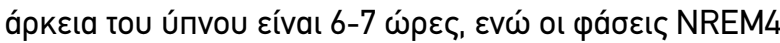

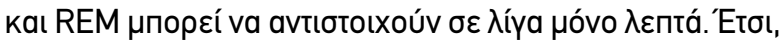

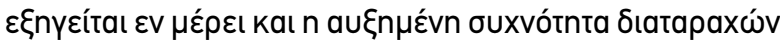

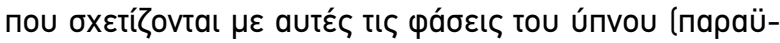

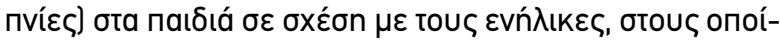

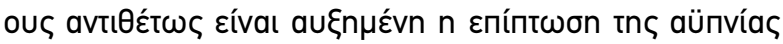

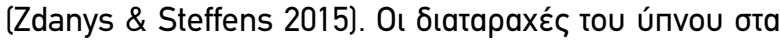

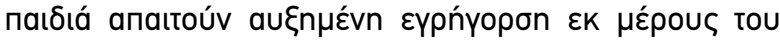

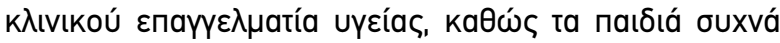

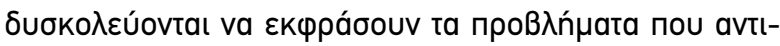

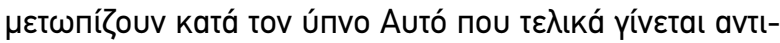

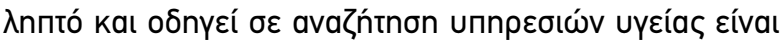

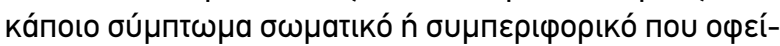

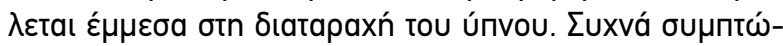

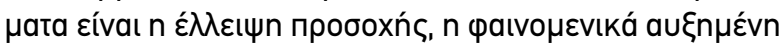

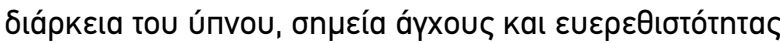

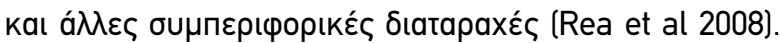

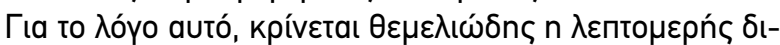

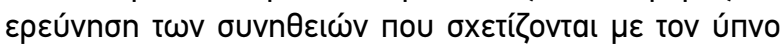

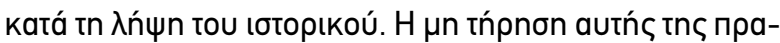

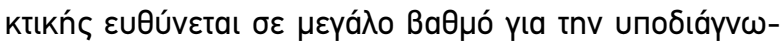

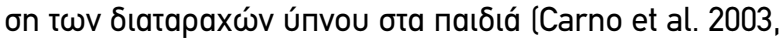
Halal \& Nunes 2014).

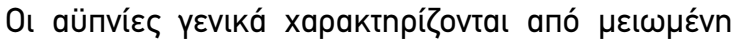

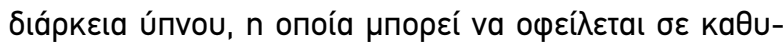

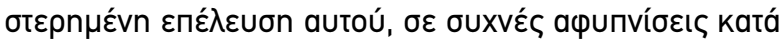

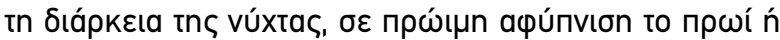

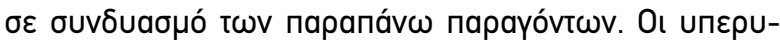

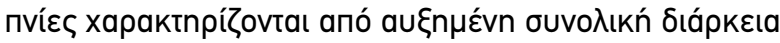

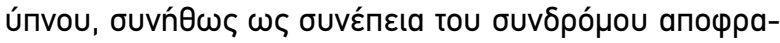

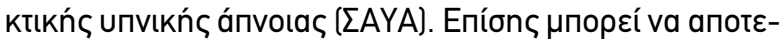

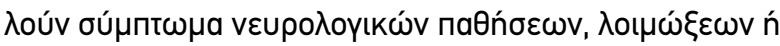

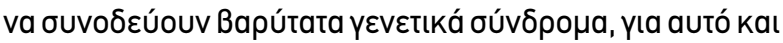

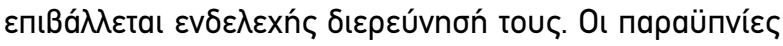

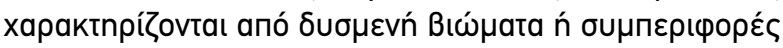

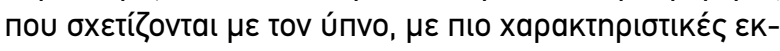

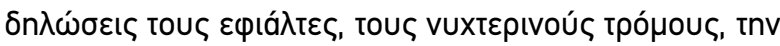

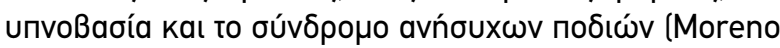

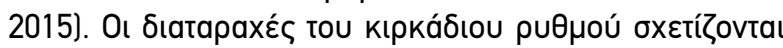

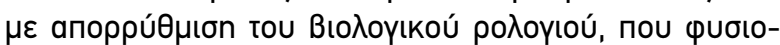

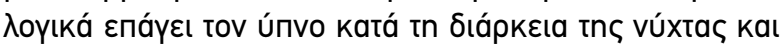

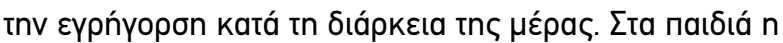

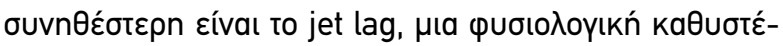




\section{REVIEW}

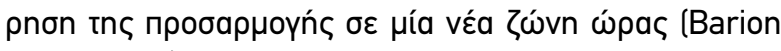

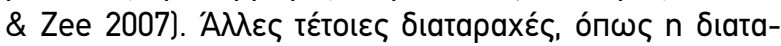

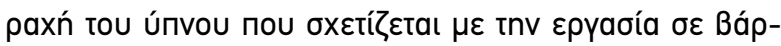

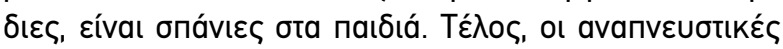

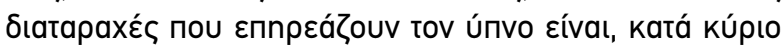

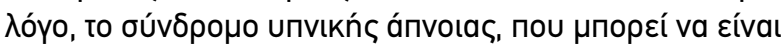

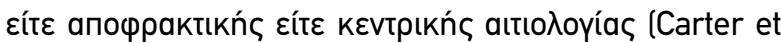

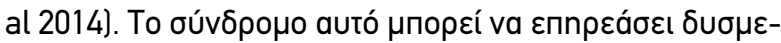

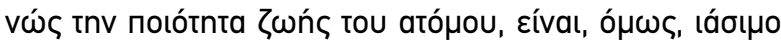

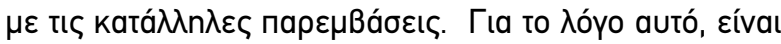

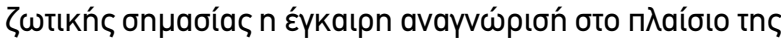

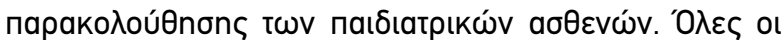

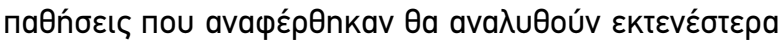
паракát $\omega$.

\section{KYPIO MEPO}

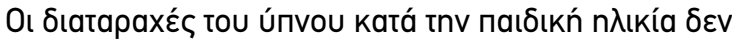

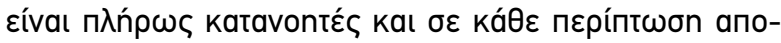

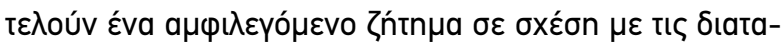

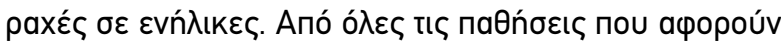

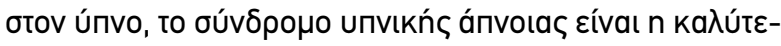

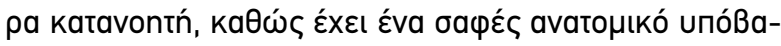

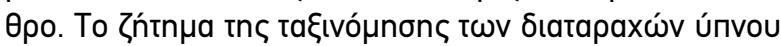

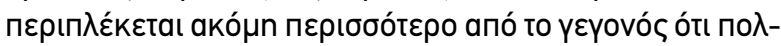

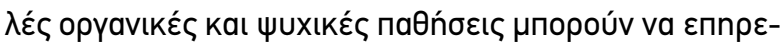

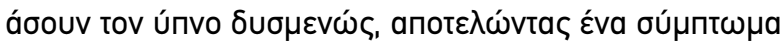

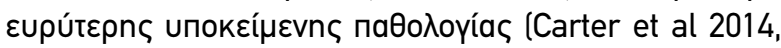
Marcus et al 2012).

\section{Aünvíєs}

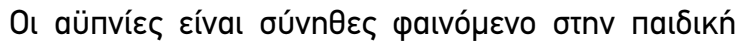

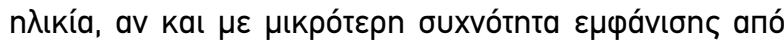

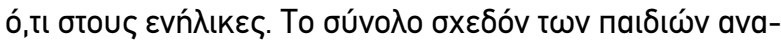

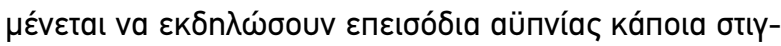

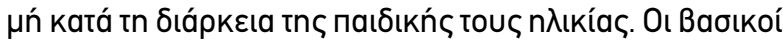

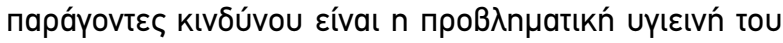

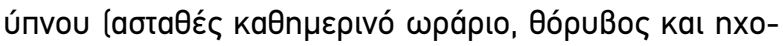

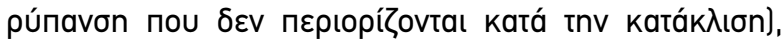

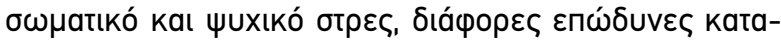

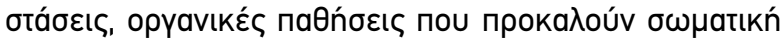

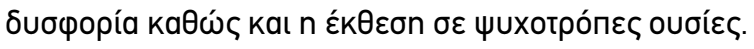

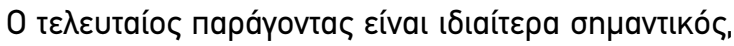

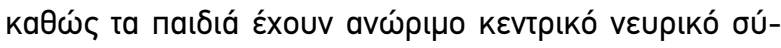

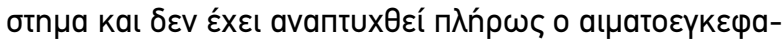

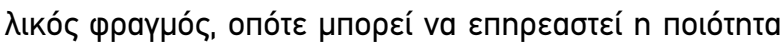

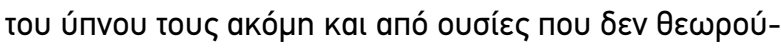

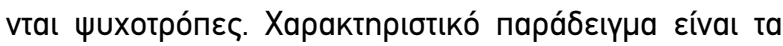

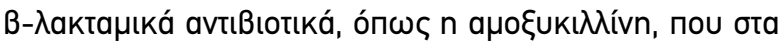

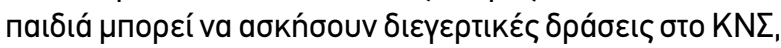

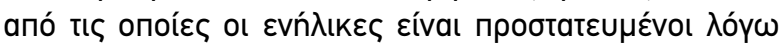

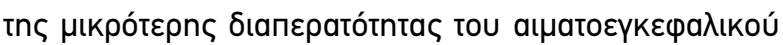

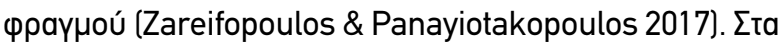

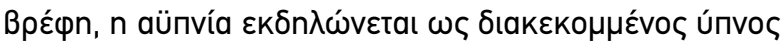

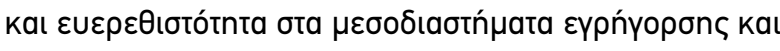

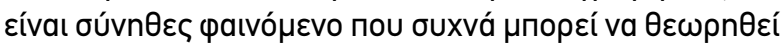

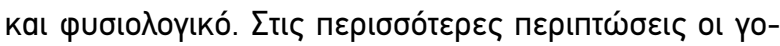

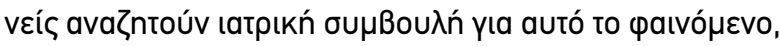

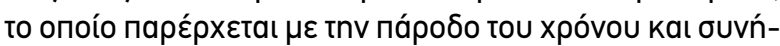

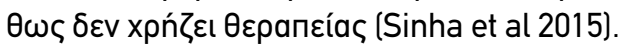

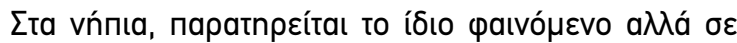

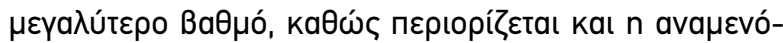

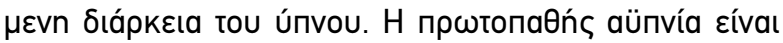

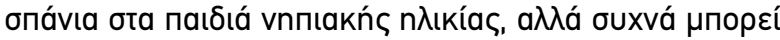

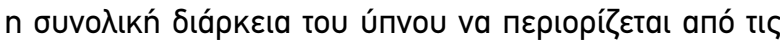

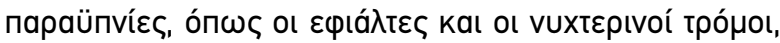

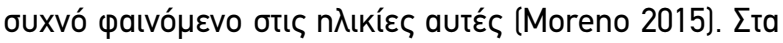

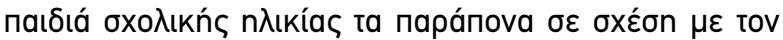

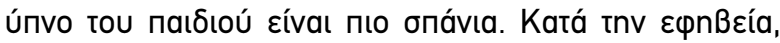

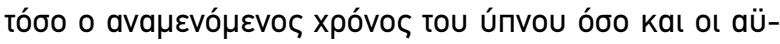

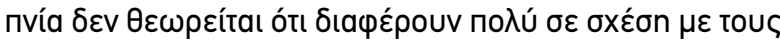

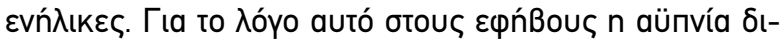

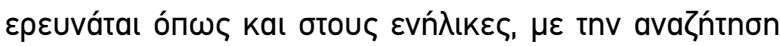

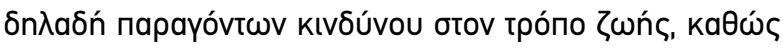

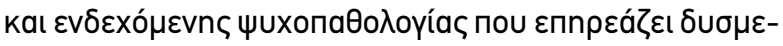

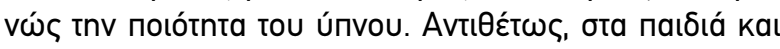

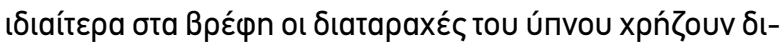

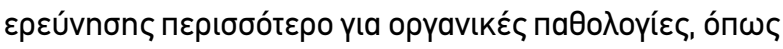

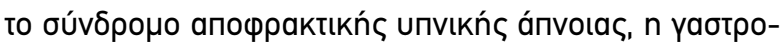

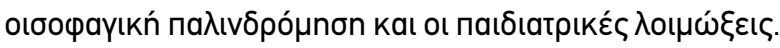

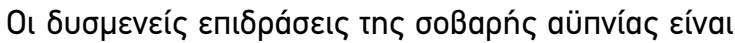

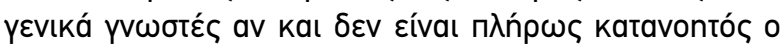

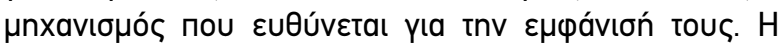

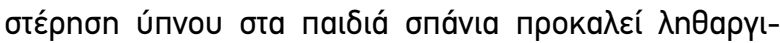

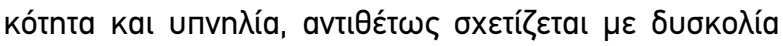

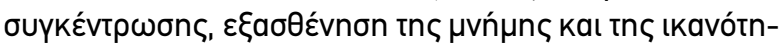

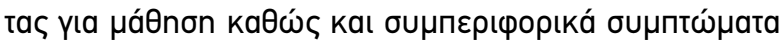

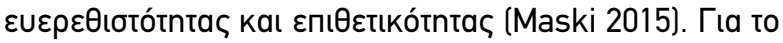

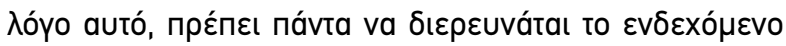

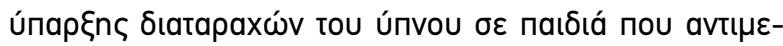

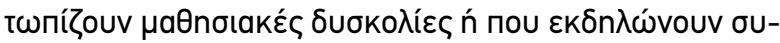

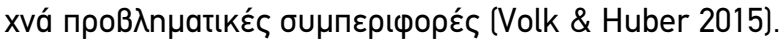

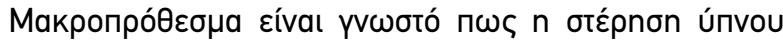

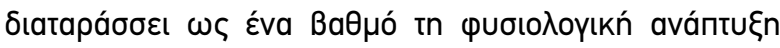

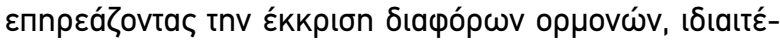

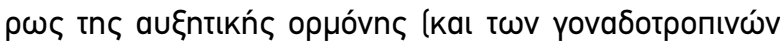

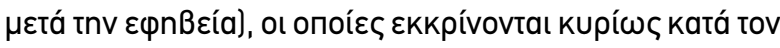

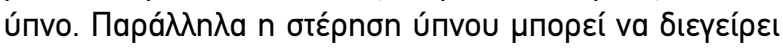

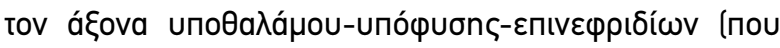

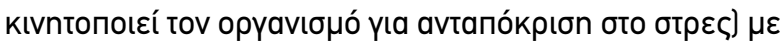

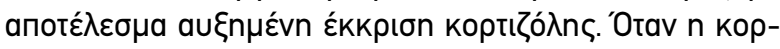

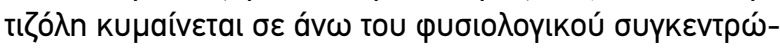

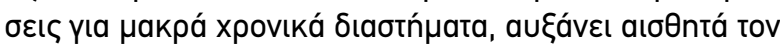

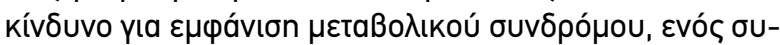

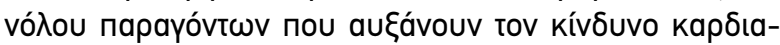

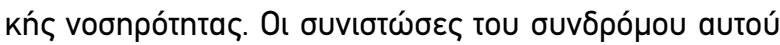

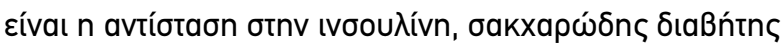




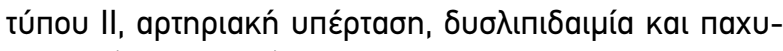
бapkía (Nevin 2013).

\section{Yпєрuпvíє}

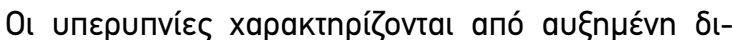

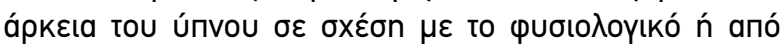

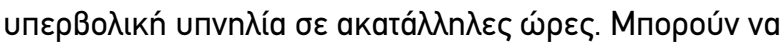

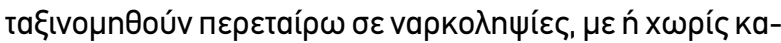

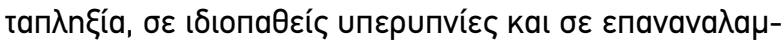

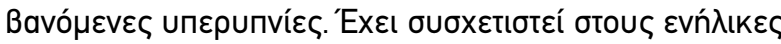

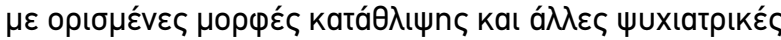

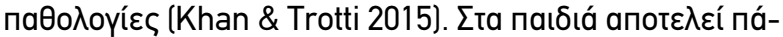

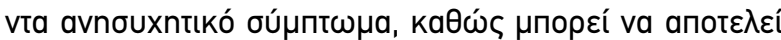

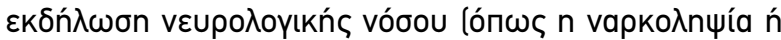

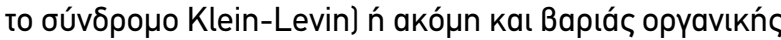

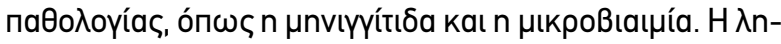

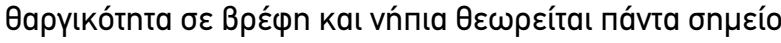
únопто үıа боßapń $\lambda$ oí $\mu \omega \xi n$ (Webb et al 2014).

\section{Параünvíєৎ}

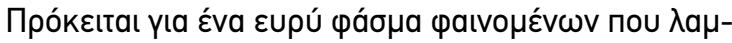

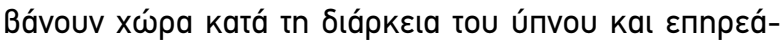

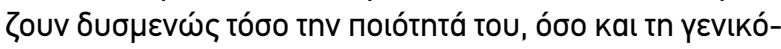

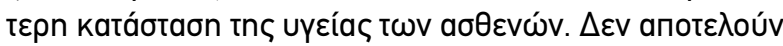

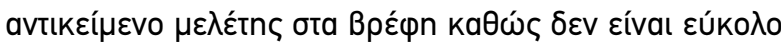

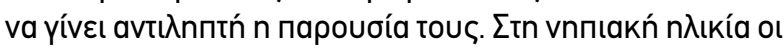

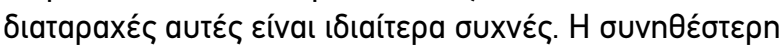

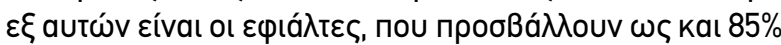

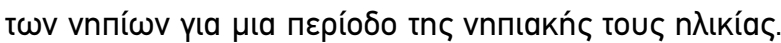

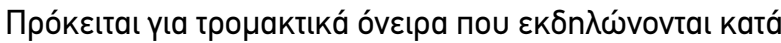

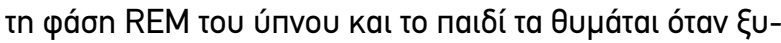

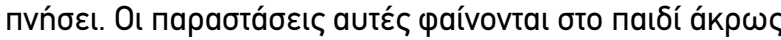

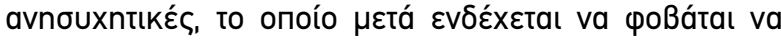

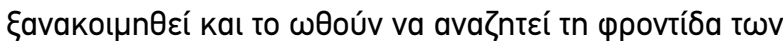

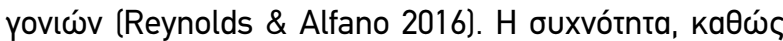

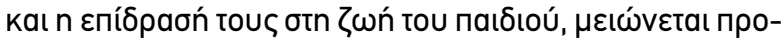

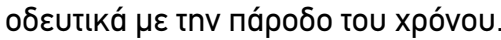

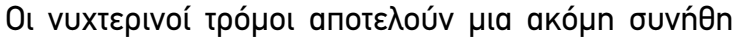

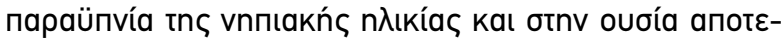

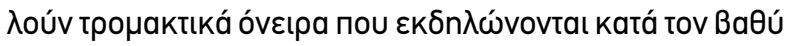

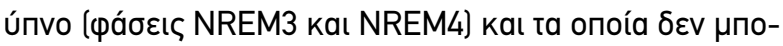

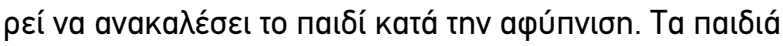

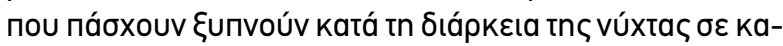

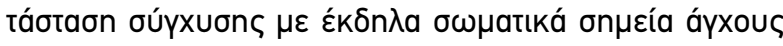

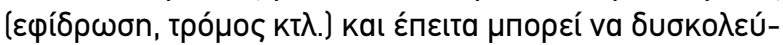

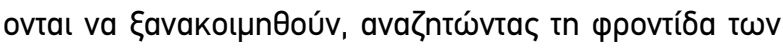

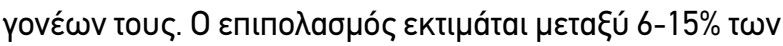

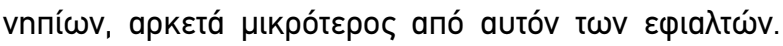

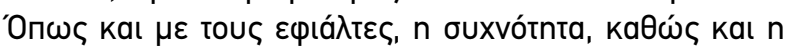

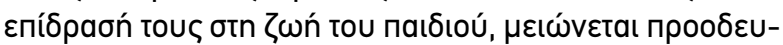
tıká $\mu \varepsilon$ tnv nápoóo tou xpóvou (Moreno 2015).

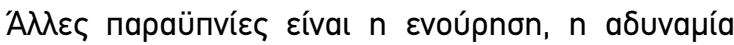

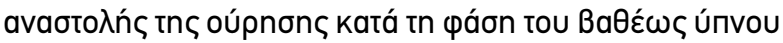

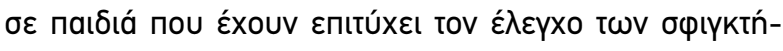

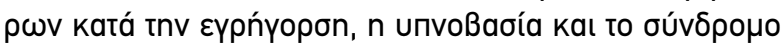

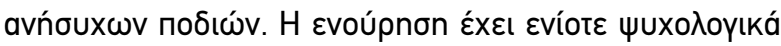

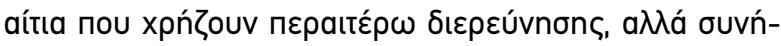

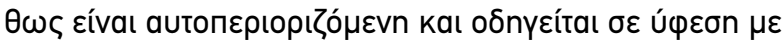

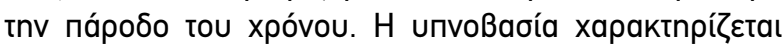

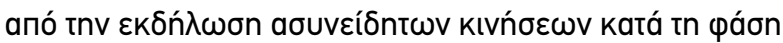

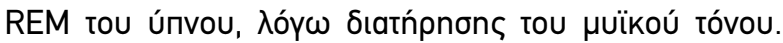

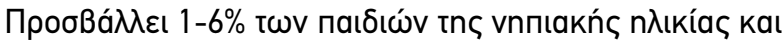

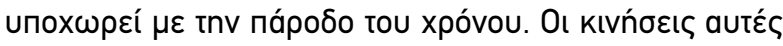

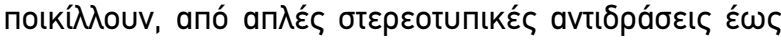

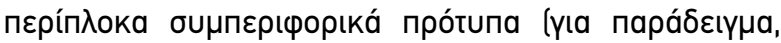

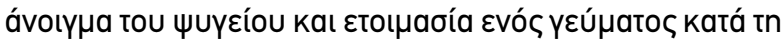

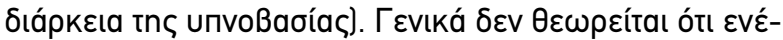

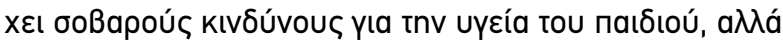

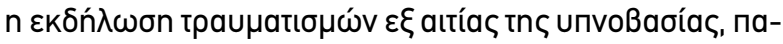

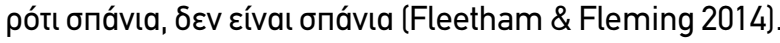

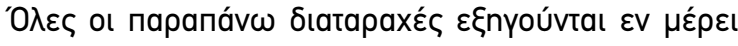

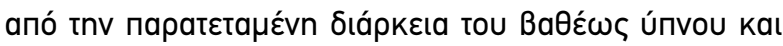

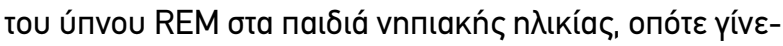

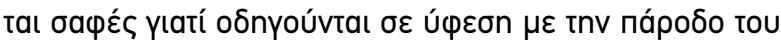

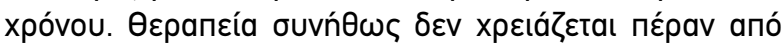

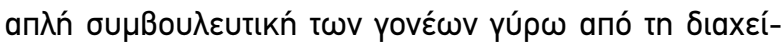

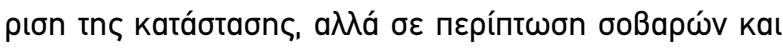

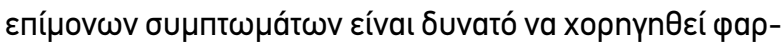

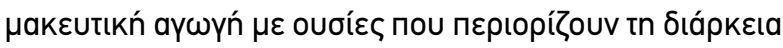

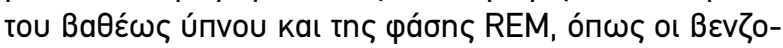

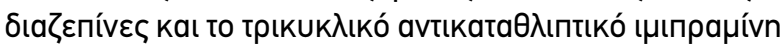
(Carter et al. 2014).

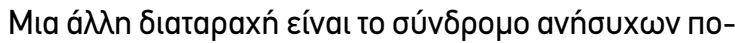

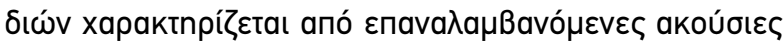

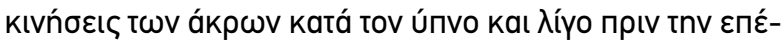

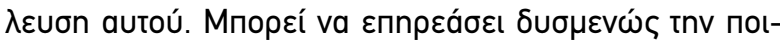

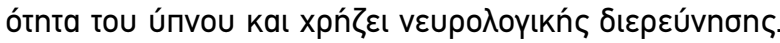

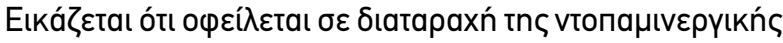

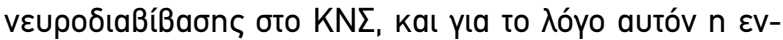

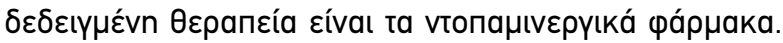

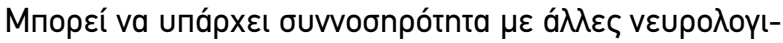

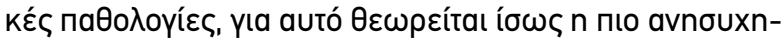
tıKń anó tıৎ naӨńoદıৎ autńc tnৎ katnyopías (Leschziner \& Gringras 2012).

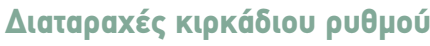

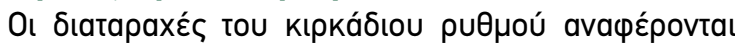

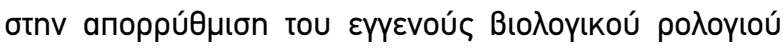

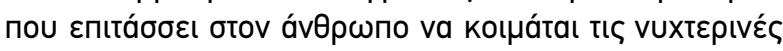

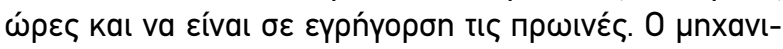

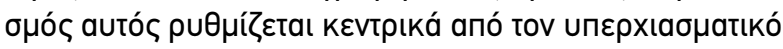

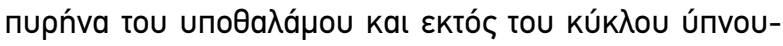

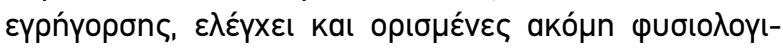

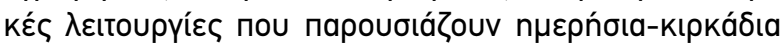

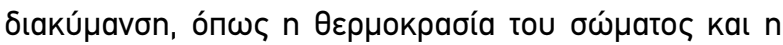

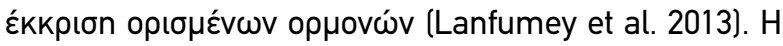




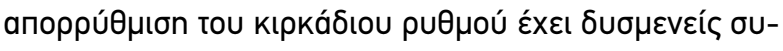

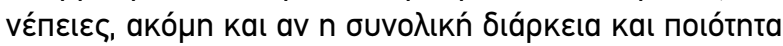

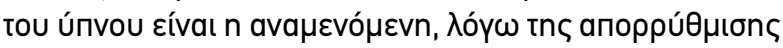

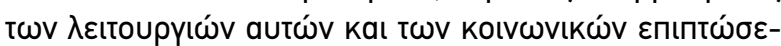

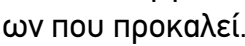

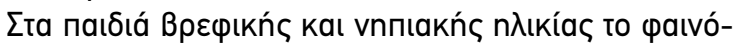

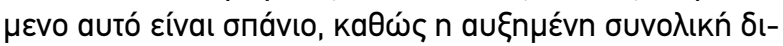

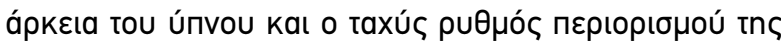

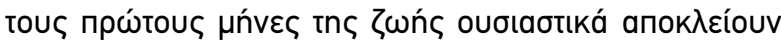

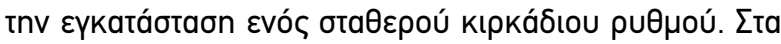

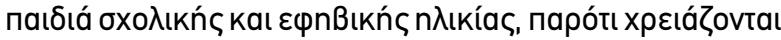

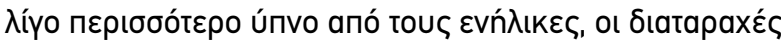

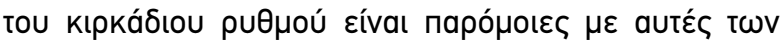

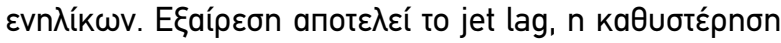

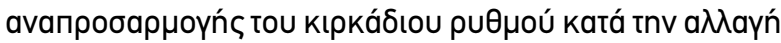

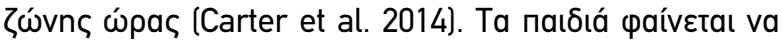

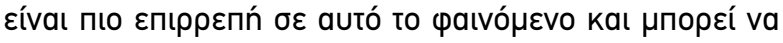

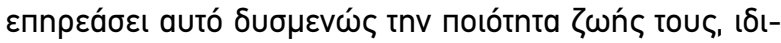

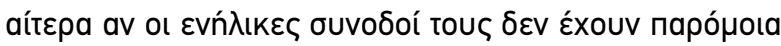

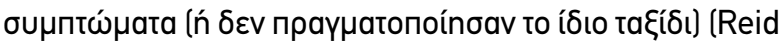
\& Abbott 2015).

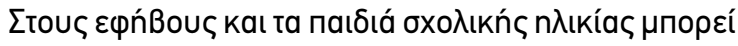

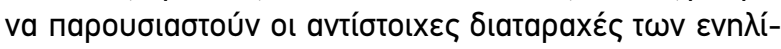

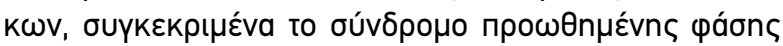

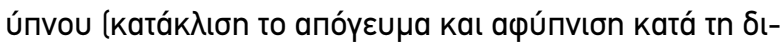

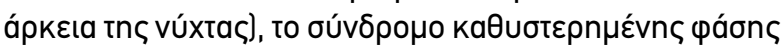

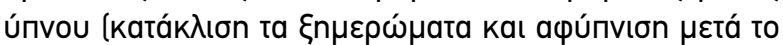

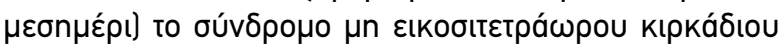

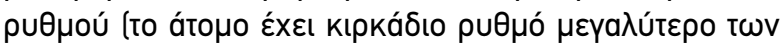

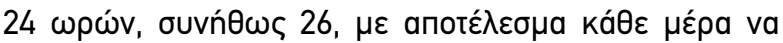

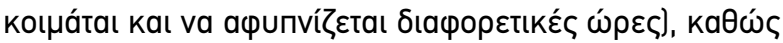

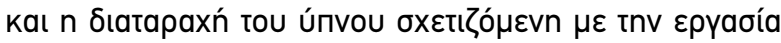

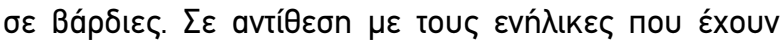

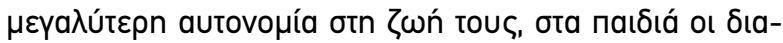

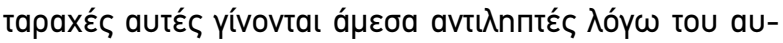

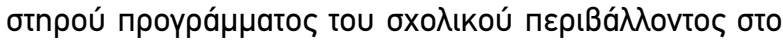

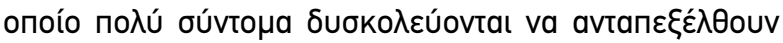

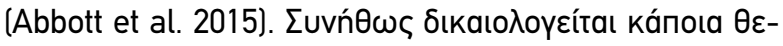

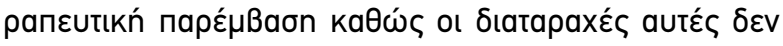

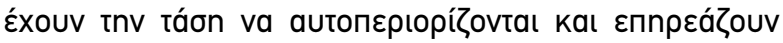

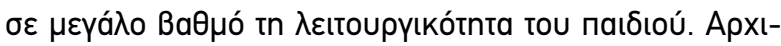

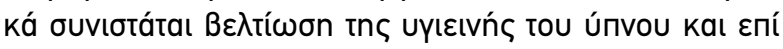

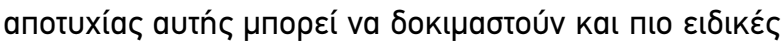

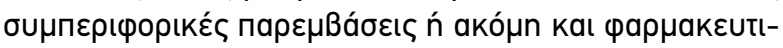

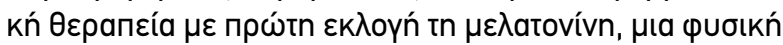

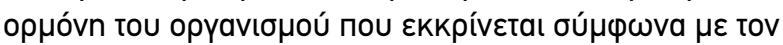

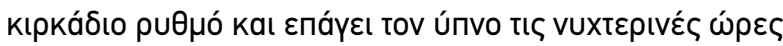

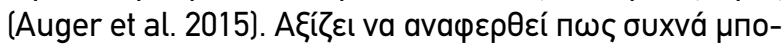

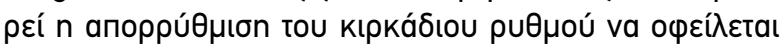

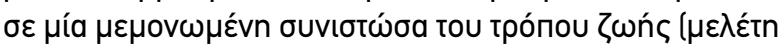

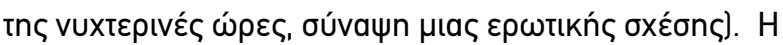

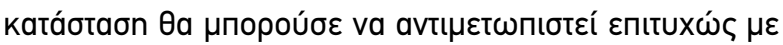

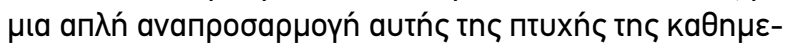

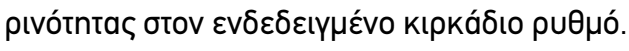

\section{¿úvôpono unvtkńs ánvotas}

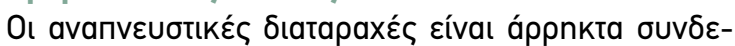

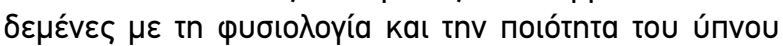

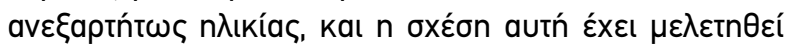

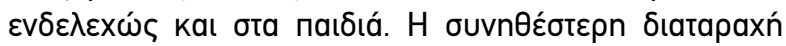

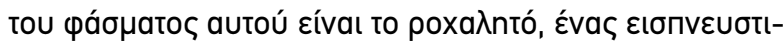

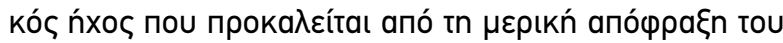

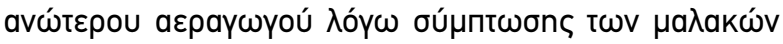

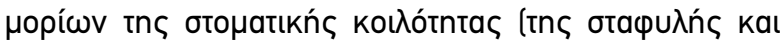
tn

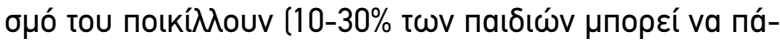

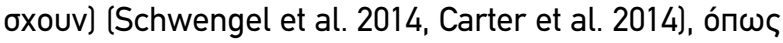

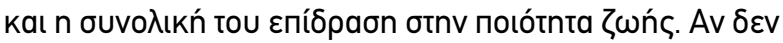

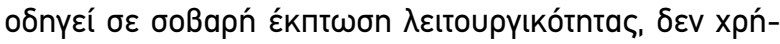

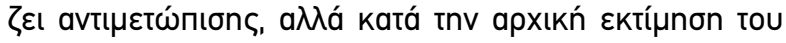

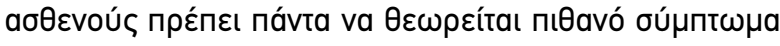

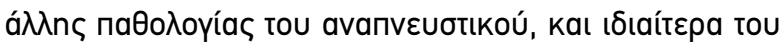

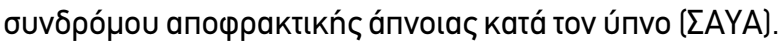

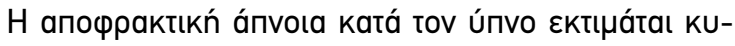

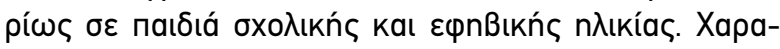

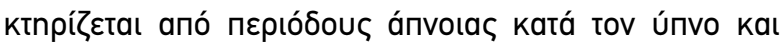

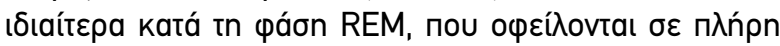

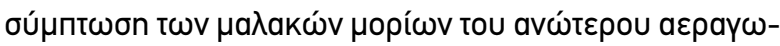

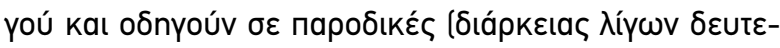

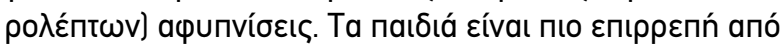

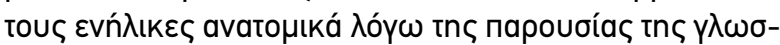

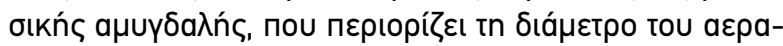

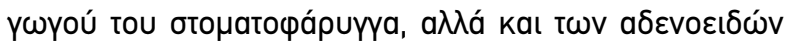

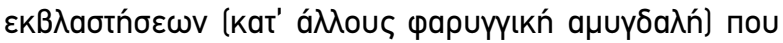

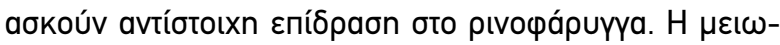

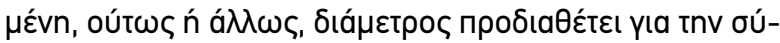

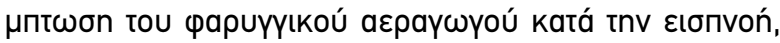

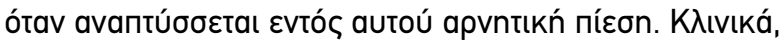

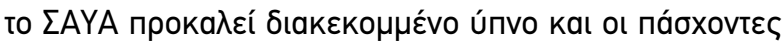

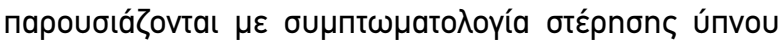

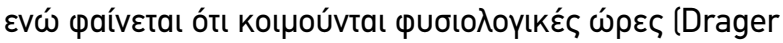

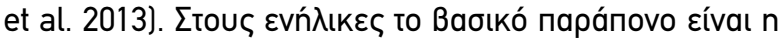

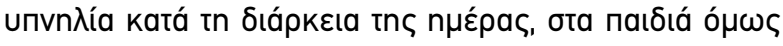

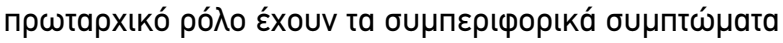

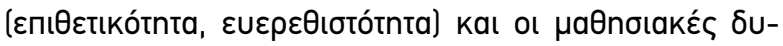

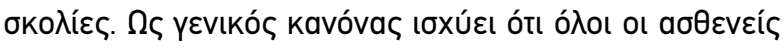

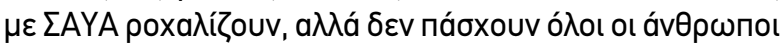

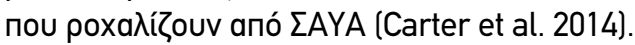

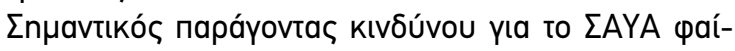

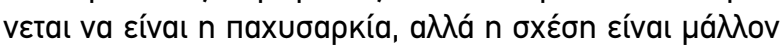

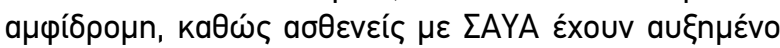

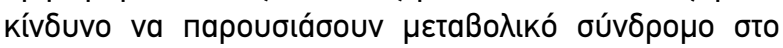

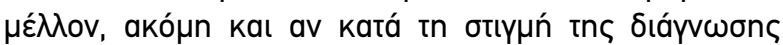

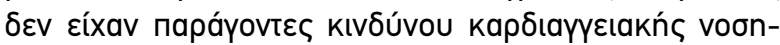
pótntas (Drager et al. 2013, Nevin 2013). Nóyw autoú

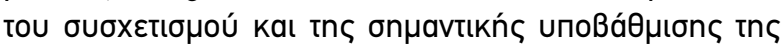




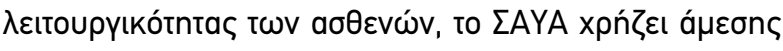

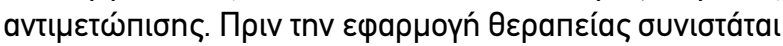

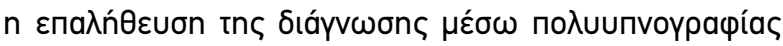

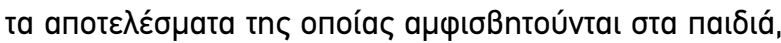
kaӨ

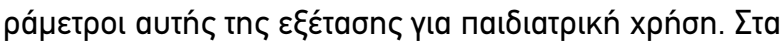

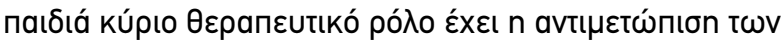

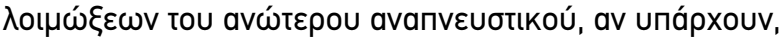

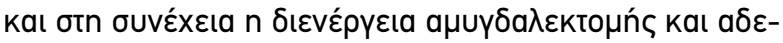

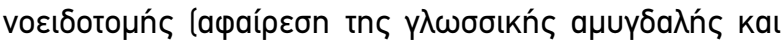

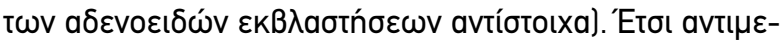

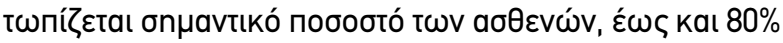

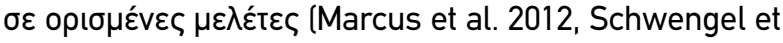
al. 2014).

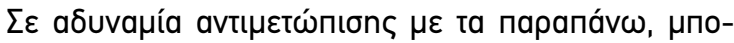

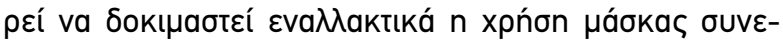

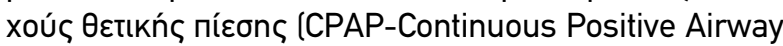

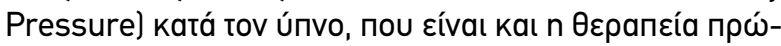

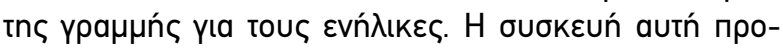

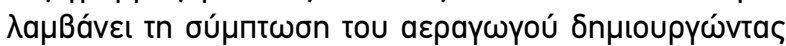

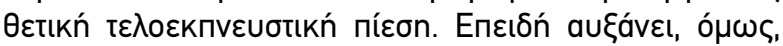

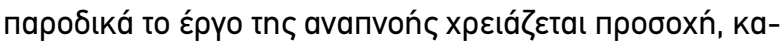

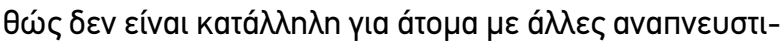

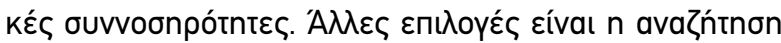

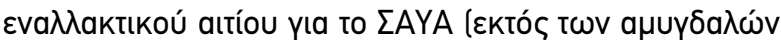

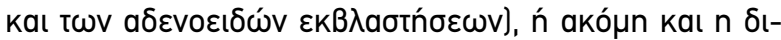

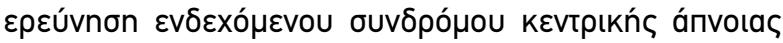

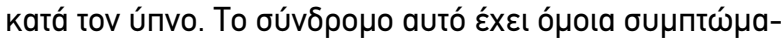

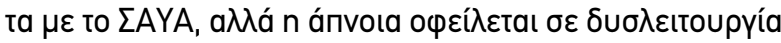

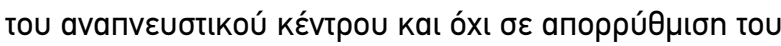

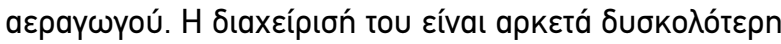

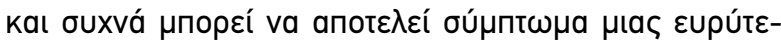

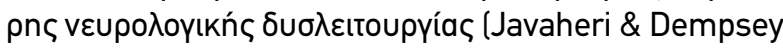
2013).

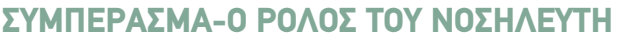

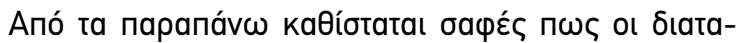

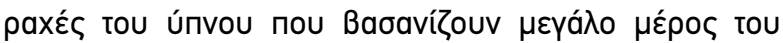

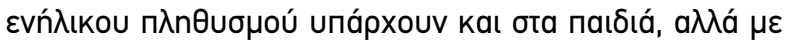

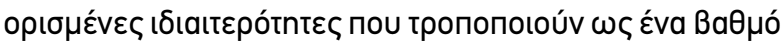

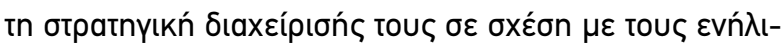

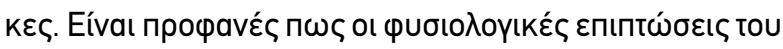

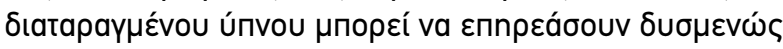

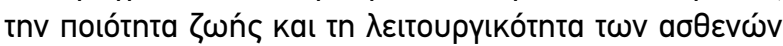

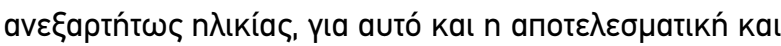

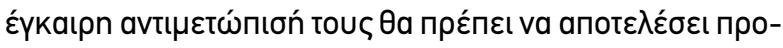

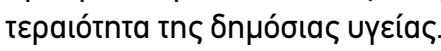

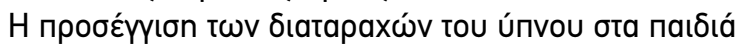

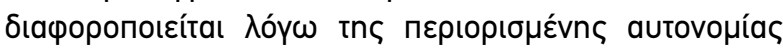

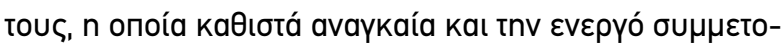

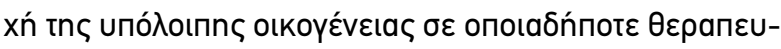

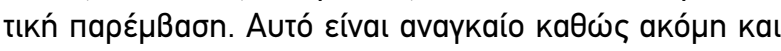

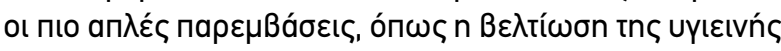
tou únvou $\delta \varepsilon v ~ \mu n o p o u ́ v ~ v a ~ \varepsilon K t \varepsilon \lambda \varepsilon \sigma \tau o u ́ v ~ a u t o ́ v o \mu a$ anó to

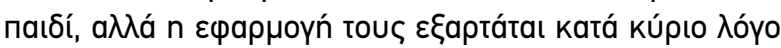

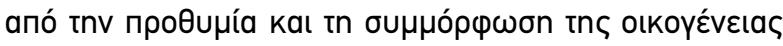

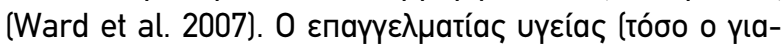

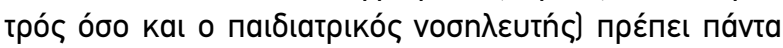

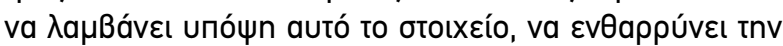

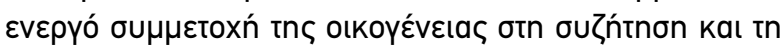

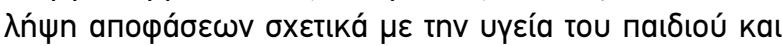

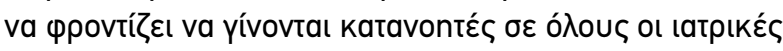

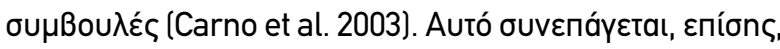

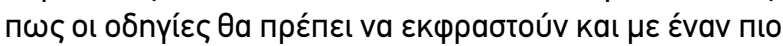

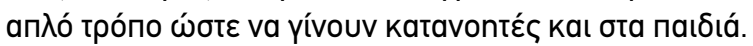

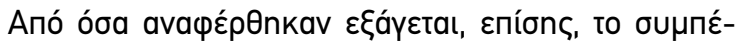

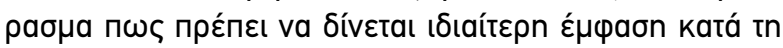

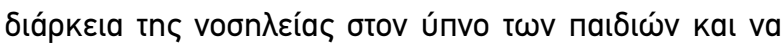

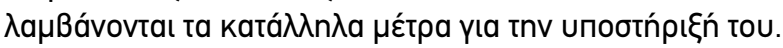

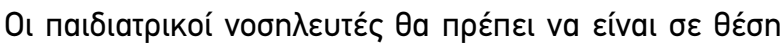

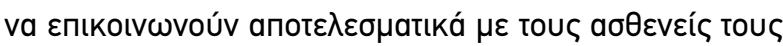

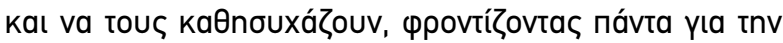

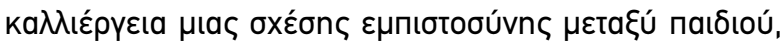

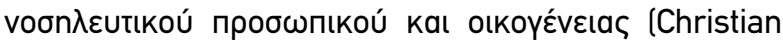

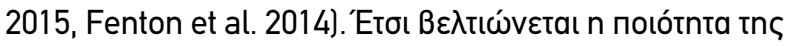

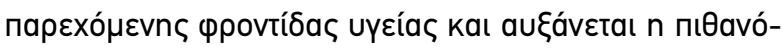

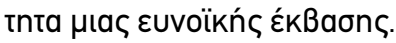




\section{BIBAIOГРАФІА}

Abbott S.M., Reid K.J. \& Zee P.C. (2015). Circadian Rhythm Sleep-Wake Disorders. The Psychiatric clinics of North America, 38(4):805-823.

Auger R.R., Burgess H.J., Emens J.S., Deriy L.V., Thomas S.M. \& Sharkey K.M. (2015). Clinical Practice Guideline for the Treatment of Intrinsic Circadian Rhythm Sleep-Wake Disorders: Advanced Sleep-Wake Phase Disorder (ASWPD), Delayed Sleep-Wake Phase Disorder (DSWPD), Non-24-Hour Sleep-Wake Rhythm Disorder (N24SWD), and Irregular Sleep-W. Journal of clinical sleep medicine: JCSM: official publication of the American Academy of Sleep Medicine 11(10):1199-1236.

Barion A. \& Zee P.C. (2007). A Clinical Approach to Circadian Rhythm Sleep Disorders. Sleep medicine 8(6):566-577.

Carno M.A., Hoffman L.A., Carcillo J.A. \& Sanders M.H. (2003). Developmental stages of sleep from birth to adolescence, common childhood sleep disorders: overview and nursing implications. Journal of pediatric nursing 18(4):274-283.

Carter K.A., Hathaway N.E. \& Lettieri C.F. (2014). Common sleep disorders in children. American family physician 89(5):368-377.

Christian B.J. (2015). Translational research: the multidimensional scope of pediatric nursing. Journal of pediatric nursing 30(1):262265.

Cirelli C. \& Tononi G. (2015). Cortical development, electroencephalogram rhythms, and the sleep/wake cycle. Biological psychiatry 77(12):1071-1078.

Drager L.F., Togeiro S.M., Polotsky V.Y. \& Lorenzi-Filho G. (2013). Obstructive sleep apnea: a cardiometabolic risk in obesity and the metabolic syndrome. Journal of the American College of Cardiology 62(7):569-576.

Fenton K., Marvicsin D. \& Danford C.A. (2014). An integrative review of sleep interventions and related clinical implications for obesity treatment in children. Journal of pediatric nursing 29(6):503-510.

Fernandez-Mendoza J., Vgontzas A.N., Calhoun S.L., Vgontzas A., Tsaoussoglou M., Gainers J., Liao D., Chrousos G.P. \& Bixler E.0. (2014). Insomnia symptoms, objective sleep duration and hypothalamic-pituitary-adrenal activity in children. European journal of clinical investigation 44(5):493-500.

Fleetham J.A. \& Fleming J.A.E. (2014). Parasomnias. CMAJ: Canadian Medical Association journal $=$ journal de l'Association medicale canadienne 186(8):E273-80.

Halal C.S.E. \& Nunes M.L. (2014). Education in children's sleep hygiene: which approaches are effective? A systematic review. Jornal de pediatria 90(5):449-456.

Javaheri S. \& Dempsey J.A. (2013). Central sleep apnea. Comprehensive Physiology 3(1):141-163.

Khan Z. \& Trotti L.M. (2015). Central Disorders of Hypersomnolence: Focus on the Narcolepsies and Idiopathic Hypersomnia. Chest
148(1):262-273

Lanfumey L., Mongeau R. \& Hamon M. (2013). Biological rhythms and melatonin in mood disorders and their treatments. Pharmacology \& therapeutics 138(2): 176-184.

Leschziner G. \& Gringras P. (2012). Restless legs syndrome. BMJ (Clinical research ed.) 344:e3056.

Marcus C.L., Brooks L.J., Draper K.A., Gozal D., Halbower A.C., Jones J., Schechter M.S., Ward S.D., Sheldon S.H., Shiffman R.N., Lehmann C. \& Spruyt K. for the American Academy of Pediatrics (2012). Diagnosis and management of childhood obstructive sleep apnea syndrome. Pediatrics 130(3):e714-55.

Maski K.P. (2015). Sleep-Dependent Memory Consolidation in Children. Seminars in pediatric neurology 22(2):130-134.

Moloney M.E., Konrad R. \& Zimmer C.R. (2011). The medicalization of sleeplessness: A public health concern. American Journal of Public Health 101(8): 1429-1433.

Moreno M.A. (2015). Sleep Terrors and Sleepwalking: Common Parasomnias of Childhood. JAMA pediatrics 169(7):704.

Nevin M.A. (2013). Pediatric obesity, metabolic syndrome, and obstructive sleep apnea syndrome. Pediatric annals 42(10):205-210.

Reid K.J. \& Abbott S.M. (2015). Jet Lag and Shift Work Disorder. Sleep medicine clinics 10(4):523-535.

Reynolds K.C. \& Alfano C.A. (2016). Things that Go Bump in the Night: Frequency and Predictors of Nightmares in Anxious and Nonanxious Children. Behavioral sleep medicine 14(4):442-456.

Schwengel D.A., Dalesio N.M. \& Stierer T.L. (2014). Pediatric obstructive sleep apnea. Anesthesiology clinics 32(1):237-261.

Sinha S., Jhaveri R. \& Banga A. (2015). Sleep Disturbances and Behavioral Disturbances in Children and Adolescents. The Psychiatric clinics of North America 38(4):705-721.

Volk C. \& Huber R. (2015). Sleep to grow smart? Archives italiennes de biologie 153(2-3):99-109.

Ward T.M., Rankin S. \& Lee K.A. (2007). Caring for children with sleep problems. Journal of pediatric nursing 22(4):283-296.

Webb T., Nugent M., Simpson P. \& Melzer-Lange M. (2014). Diagnostic findings in infants presenting to a pediatric emergency department for lethargy or feeding complaints. Pediatric emergency care 30(3):151-156.

Zareifopoulos N. \& Panayiotakopoulos G. (2017). Neuropsychiatric Effects of Antimicrobial Agents. Clinical Drug Investigation 37(5):423-437.

Zareifopoulos N. \& Papatheodoropoulos C. (2016). Effects of 5-HT7 receptor ligands on memory and cognition. Neurobiology of learning and memory 136:204-209.

Zdanys K.F. \& Steffens D.C. (2015). Sleep Disturbances in the Elderly. The Psychiatric clinics of North America 38(4):723-741. 


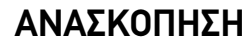

\title{
Sleep disorders in childhood
}

\author{
Alexandra-Maria Kazala', Vasiliki Karagianni', George Grammatikas² \\ 1. Nurse \\ 2. RN, MSc - Physiotherapist, Patras General Children's Hospital "Karamandanio"
}

\section{ABSTRACT}

Sleep disorders have reached epidemic rates in the modern world. It is important to note that they can affect children as well as adults. Sleep is of the utmost importance for the maintenance of body homeostasis. Many disease processes affecting sleep have been associated with numerous symptoms, including cognitive impairment, fatigue as well as a wide range of behavioral, hormonal and metabolic derangements. Sleep disorders in childhood can be classified by age of incidence (in infants, toddlers, school-age children or adolescents) and by the exact nature of the symptoms. They are classified in insomnias, hypersomnias, parasomnias, circadian rhythm disorders and respiratory disorders which affect sleep. Parasomnias are the most common sleep disorders in toddlers, with night terrors, nightmares and sleepwalking being the most common. Respiratory disorders are the most important in older children, especially obstructive sleep apnea syndrome. All sleep disorders are clinically manifested with symptoms of sleep deprivation. Their management always involves improvement of sleep hygiene, though in some cases (such as obstructive sleep apnea) specific medical treatment is also available and warranted. A three-way relationship of trust, compassion and cooperation between child, family and healthcare professional is a prerequisite for the effective management of childhood sleep disorders. Nursing staff are the main factor cultivating that relationship in the context of inpatient care.

Keywords: Circadian rhythm, Nursing, Obstructive sleep apnea syndrome, Pediatrics, Sleep disorders 